\title{
Acinetobacter baumannii: biology and drug resistance - role of carbapenemases
}

\author{
Pawel Nowak, Paulina Paluchowska
}

\author{
Department of Pharmaceutical Microbiology, Faculty of Pharmacy, Jagiellonian University \\ Medical College, Krakow, Poland
}

\begin{abstract}
Acinetobacter baumannii is a Gram-negative, glucose-non-fermenting, oxidase-negative coccobacillus, most commonly associated with the hospital settings. The ability to survive in adverse environmental conditions as well as high level of natural and acquired antimicrobial resistance make $A$. baumannii one of the most important nosocomial pathogens. While carbapenems have long been considered as antimicrobials of last-resort, the rates of clinical A. baumannii strains resistant to these antibiotics are increasing worldwide. Carbapenem resistance among $A$. baumannii is conferred by coexisting mechanisms including: decrease in permeability of the outer membrane, efflux pumps, production of beta-lactamases, and modification of penicillin-binding proteins. The most prevalent mechanism of carbapenem resistance among $A$. baumannii is associated with carbapenem-hydrolysing enzymes that belong to Ambler class D and B beta-lactamases. In addition, there have also been reports of resistance mediated by selected Ambler class A carbapenemases among $A$. baumannii strains. Resistance determinants in $A$. baumannii are located on chromosome and plasmids, while acquisition of new mechanisms can be mediated by insertion sequences, integrons, transposons, and plasmids. Clinical relevance of carbapenem resistance among strains isolated from infected patients, carriers and hospital environment underlines the need for carbapenemase screening. Currently available methods vary in principle, accuracy and efficiency. The techniques that deserve particular attention belong to both easily accessible unsophisticated methods as well as advanced techniques based on mass spectrometry or molecular biology. While carbapenemases limit the therapeutic options in A. baumannii infections, studies concerning novel beta-lactamase inhibitors offer a new insight into effective therapy. (Folia Histochemica et Cytobiologica 2016, Vol. 54, No. 2, 61-74)
\end{abstract}

Key words: Acinetobacter baumannii; virulence factors; drug resistance; carbapenems; carbapenemases; metallo-beta-lactamases; oxacillinases; CHDL; resistance detection; carbapenemase inhibitors

\section{Introduction}

Acinetobacter baumannii is a Gram-negative, nonmotile, glucose-non-fermenting oxidase-negative and increasingly important opportunistic pathogen. The bacterium can survive on solid and dry surfaces up to 5 months, which is attributed to: simplicity of its nutritional requirements, ability to grow in the wide

Correspondence address: P. Nowak, Pharm.D.

Department of Pharmaceutical Microbiology,

Faculty of Pharmacy

Jagiellonian University Medical College

Medyczna 9, 30-688 Krakow, Poland

tel.: +48 1262057 52, fax: +48 126205758

e-mail: p.nowak@uj.edu.pl range of temperatures and $\mathrm{pH}$ values, high degree of resistance to disinfectants and antiseptics, ability to form biofilm on abiotic substrata (environmental surfaces or medical devices, such as catheters or respiratory equipment) as well as biotic surfaces [1-5]. These features are likely to be a major contributing factor to nosocomial spread of the $A$. baumannii [5]. It is thought that $A$. baumannii infections are mostly acquired after exposure to contaminated hospital equipment or by direct contact with healthcare personnel that have been previously exposed to the microorganism [6]. The other important features responsible for $A$. baumannii clinical relevance are: high natural resistance as well as an outstanding ability to up-regulate innate and acquire foreign mechanisms of antimicrobial resistance [5]. 


\section{Clinical relevance}

Since the 1980s the clinical importance of $A$. baumannii has radically emerged [7]. The increase of incidence of $A$. baumannii infections is believed to be attributed to advancements in medical procedures, which may result in rise of susceptible population of patients [8]. It was also noted that its antimicrobial resistance has progressively increased since the 1970s [7]. Consequently, $A$. baumannii has been described by Infectious Diseases Society of America as one of the most significant hospital pathogens [9]. The genus Acinetobacter belongs to Moraxellaceae family and consists of at least 21 described species, with A. baumannii as the most clinically relevant [10]. While Acinetobacter spp. phenotypical identification system was described, it does not distinguish all known so far Acinetobacter species [11]. Current Acinetobacter spp. taxonomy comprise $A$. calcoaceticus $-A$. baumannii complex (Abc) which includes: $A$. calcoaceticus (genomic species 1), A. baumannii (genomic species 2), $A$. pittii (genomic species 3 ) and $A$. nosocomialis (genomic species 13TU), that are all highly genetically related and difficult to distinguish phenotypically [10]. It is considered that more reliable methods of identification are represented by amplified 16S rRNA gene restriction analysis (ARDRA) and amplified fragment length polymorphism (AFLP) [12]. Acinetobacter baumannii can cause numerous acute hospital infections represented by: respiratory infections (in particular ventilator-associated pneumonia; VAP), bloodstream infections, urinary tract infections, skin and soft tissue infections, burn and surgical wound infections, endocarditis, meningitis, and osteomyelitis [7]. The risk factors that make patients prone to the colonisation and infection with $A$. baumannii comprise: considerable surgical procedures, major trauma, premature or old age, hospitalisation, antimicrobial therapy as well as medical treatment including mechanical ventilation, intravascular catheters, urinary catheters and drainage tubes [11]. This bacterium is reported as responsible for $2-10 \%$ of all Gram-negative hospital infections, mainly affecting critically ill patients, particularly hospitalised in intensive care units [7]. Furthermore, A. baumannii healthcare-associated infections may increase the risk of mortality from $8 \%$ to $40 \%$ [13]. The multicentre studies conducted by Hidron and co-workers revealed the growing occurrence of A. baumannii nosocomial infections, with $8.4 \%$ of VAP, $2.2 \%$ of central line-associated bloodstream infections, $1.2 \%$ of catheter-associated urinary tract infections and $0.6 \%$ of surgical site infections [14]. Despite the nosocomial infections, $A$. baumannii can also induce community-acquired infections (CA-Ab
- community-acquired $A$. baumannii), which are reported predominantly in tropical regions of the world [15]. Pneumonia and bacteraemia are the most prevalent clinical syndromes related to $A$. baumannii infection in these settings. Community acquired pneumonia affects particularly individuals with underlying chronic obstructive pulmonary disease, renal failure, or diabetes mellitus, as well as heavy smokers or alcoholics [8]. As described by Dexter and co-authors $\mathrm{CA}-\mathrm{Ab}$ pneumonia is characterised with fulminant course associated with a rapid onset of fever, acute respiratory symptoms and multi-organ dysfunction, with high mortality rate representing $64 \%$ [16]. Despite the hospital and community acquired $A$. baumannii infections, its occurrence appeared to be also related to injuries in war conflicts in e.g. Afghanistan, Iraq and Syria $[17,18]$ as well as natural disasters, such as earthquakes and tsunami [19, 20]. Epidemic spread of $A$. baumannii in above-mentioned extreme situations is attributed by some authors to substantial pressure on hospital emergency wards, which may result in failure in infection control precautions [7].

\section{Virulence factors}

Till date, there have been only a few virulence factors (VF) described in A. baumannii. Recent analysis concerning genome sequencing, genetic manipulations and applications of animal models allow believing that current knowledge regarding additional factors will be broadened. Among virulence determinants responsible for the A. baumannii pathogenicity, there may be mentioned: lipopolysaccharide (LPS), capsular polysaccharides (CPS), A. baumannii outer membrane protein A (AbOmpA), outer membrane vesicles (OMV), phospholipase D (PLD), and biofilm $[6,21]$.

The studies concerning the role of $A$. baumannii LPS indicated the contribution of the surface carbohydrates residues in the virulence [22]. The endotoxins are also considered as a potent stimulators of inflammatory signalling in human monocytic cells, dependent on both TLR2 (toll-like receptor) and TLR4 receptors. Therefore, the pathology of Acinetobacter infections may be associated with exaggerated innate immune response to the LPS [23-25]. Further determinants implicated in the virulence of A. baumannii are capsular polysaccharides. Studies conducted by Russo and co-workers revealed that K1 capsule acquire from clinical isolate of $A$. baumannii AB307-0294 appeared to play an important role in in vivo as well as in vitro bacterial protection. The active protection of the CPS enables $A$. baumannii to avoid bactericidal activity of the complement. The authors 
also suggested that the CPS protection against the effects of phagocytes or antimicrobial peptides requires further studies [26]. AbOmpA is considered to be one of the best-characterised $A$. baumannii virulence factors. It is responsible for the damage to the human airway cells via induction of the apoptosis. This process is mediated by the release of proapoptotic molecule cytochrome $\mathrm{c}$ and the apoptosis-inducing factor. Moreover, AbOmpA is believed to play a role in adherence, invasion of epithelial cells and may contribute to the dissemination of $A$. baumannii during infection [27, 28]. Furthermore, above-mentioned protein is also implicated in the surface motility, resistance to complement and biofilm formation $[6,21]$. Acinetobacter baumannii as well as some other Gram-negative bacteria secrete outer membrane vesicles. OMVs have been reported to take part in delivering virulence factors to the interior of the host cells, facilitating horizontal gene transfer, and protecting bacterial cells from the host immune response [29]. Proteomic analysis of $A$. baumannii OMVs revealed the presence of more than 130 proteinsincluding: AbOmpA, serine- as well as $\mathrm{Zn}$-dependent proteases, phospholipases, bacterioferritin, $\mathrm{Cu} / \mathrm{Zn}$ superoxide dismutase, catalase, and ferrichrome-iron receptor [30]. Further data published by Jun and co-workers demonstrated considerable role of $A$. baumannii OMVs in stimulation of innate immune response that may contribute to immunopathology of the infected host [31]. Another group of $A$. baumannii $\mathrm{VF}$ include phospholipases. These enzymes are associated with cleavage of the host cells phospholipids, therefore promoting the bacterial invasion [6]. Studies concerning the role of $A$. baumannii phospholipase $\mathrm{D}$ in murine model of pneumonia revealed that disruption of PLD results with reduction of the bacterial survival in serum, deficiency in epithelial cell invasion and diminished pathogenesis [32].

Biofilm is a multicellular complex with three-dimensional structure, relevant in the infection. While its formation comprises the surface of the host cells and indwelling medical devices, it plays an essential role in the pathogenesis. Moreover, the presence of biofilm reduces the antibiotic penetration, leading to the development of drug resistance [33-37]. The CsuA/BABCDE usher-chaperone pili assembly system has been involved in $A$. baumannii biofilm organization on abiotic surfaces [38]. The expression level of $c s u$ operon is regulated by the two-component system - BfmS/BfmR, comprising a sensor kinase and a response regulator [39]. Biofilm-associated protein (Bap), expressed on the surface of the bacterial cells, is implicated in cell-to-cell adhesion providing biofilm development and maturation on different substrata [40]. Furthermore, one of the most important components of exopolysaccharides constituting biofilm matrix was polysaccharide polymer poly-beta-1,6- $N$-acetylglucosamine (PNAG) that is crucial for maintaining the integrity of $A$. baumannii biofilm under nutrient limitation and other environmental stresses [41]. As mentioned above, the outer membrane protein OmpA (38 kDa) plays an important role in the pathogen attachment to the human cells and Candida filaments, as well as in biofilm development on plastics [42]. Another factor implicated in A. baumannii biofilm formation on abiotic surfaces is represented by 3-hydroxy-C12-HSL molecule, encoded by the $a b a I$ autoinducer synthase gene [43].

\section{Drug resistance}

Acinetobacter baumannii is characterised by high level of intrinsic resistance to many groups of antimicrobials (e.g. glycopeptides, macrolides, lincosamides, and streptogramins) [44]. Moreover, this bacterium is able to develop resistance to all classes of antimicrobial agents used in the therapy. The process can be associated with genetic changes leading to membrane alterations, overexpression of efflux pumps (EP), overexpression of intrinsic antibiotic modifying enzymes, modifications of target sites for antimicrobial agents, and acquisition of novel resistance determinants. Acinetobacter baumannii strains enhanced by selective pressure of hospital environment may gain resistance via mutational changes as well as by horizontal gene transfer from other members of the species, genus, non-fermenters and/or Enterobacteriaceae family. Observed for a period of time, rapid accumulation of resistance determinants to multiple classes of antimicrobials among $A$. baumannii strains resulted in the elimination of penicillins, cephalosporins, aminoglycosides, quinolones, and tetracyclines as effective treatment options for many clinical isolates. Consequently, due to their good activity and low toxicity, carbapenems have left as one of the major therapeutic options in A. baumannii infections [9].

\section{Carbapenems}

Carbapenems are considered to play an essential role in antimicrobial therapy worldwide. Regarding antibacterial activity, they demonstrate the broadest spectrum among beta-lactam antibiotics. Therefore, carbapenems are often administered as "antibiotics of last-resort" or "last-line agents" in critically ill patients in the treatment a variety of infection. 


\section{Chemistry}

The carbapenems differ from penicillins by the presence of carbon instead of sulphur in the position C-1 and possession of double bond between C-2 and C-3 in five-membered ring. The broad spectrum of antimicrobial agents is attributed to the natural resistance to different groups of beta-lactamases. In comparison to penicillins and cephalosporins, carbapenems possess trans-alpha-1-hydroxyethyl substituent at the 6 position, which results in high stability to beta-lactamases. The first described carbapenem was thienamycin, however the unstable nature of this compound resulted with the development of an $N$-formimidoyl derivative named imipenem. Nevertheless, imipenem as well as panipenem are degraded in brush border of renal tubules by dehydropeptidase-1 (DHP-1), forcing the co-administration of cilastatin or betamiprion, respectively [45]. Following carbapenems, such as meropenem, ertapenem, biapenem and doripenem, due to the presence of a methyl group in the 1-beta position, are not affected by DHP-1 inhibition. Meropenem as a derivative of thienamycin has a pyrrolidinyl substituent at 2 position, which is thought to provide the enhanced Gram-negative activity. Doripenem is a synthetic 1-beta-methylcarbapenem, differing from meropenem with the presence of sufamoylaminomethyl group. This modification increases doripenem activity towards non-fermentative Gram-negative bacilli $[45,46]$. Another carbapenem, introduced to the therapy in 2001, is ertapenem. This broad-spectrum beta-lactam differs structurally from meropenem in a meta-substituted benzoic acid group at the 2 binding position. In consequence, increasing of the molecular weight and lipophilicity of the molecule was observed. Structural changes of the molecule also resulted in the increased half-life and decreased permeation through the Gram-negative bacteria cell wall [47].

\section{Mechanism of action}

The mode of action of carbapenems as well as other beta-lactam antibiotics is associated with the structure and biosynthesis of the bacterial cell wall [48]. Carbapenems enter the Gram-negative bacteria via outer membrane proteins (also described as porins), cross the periplasmic space and acetylate the penicillin-binding proteins (PBPs). PBPs are described as enzymes (i.e. peptidases, transglycosylases) responsible for the formation of peptidoglycan in the bacterial cell wall. Therefore, the bactericidal activity of carbapenems is associated with binding to PBP and, in consequence, the inhibition of peptide cross-linking and other peptidase reactions. Carbapenem binding of PBP leads to weakening of the peptidoglycan and may result with the cell burst due to the osmotic pressure. Furthermore, one of the essential features of the carbapenems is the ability to bind to multiple different PBPs [46].

Imipenem binds favourably to PBP2 and PBP1, while possess weak affinity towards PBP3. Meropenem affinity for the various PBPs differs between Gram-positive and Gram-negative bacteria. Among Gram-negative bacteria, this carbapenem binds preferentially to PBPs 2, 3 and 4, as well as exhibits strong affinity to PBPs $1 \mathrm{a}$ and $1 \mathrm{~b}$. Ertapenem binds primarily to PBP2 of Escherichia coli than PBP3, and has good affinity for PBP1a, 1b, 4 and 5. Doripenem has high affinity towards various PBPs exhibited by many bacterial species. This carbapenem presents high affinity for PBP2 and PBP3 in Pseudomonas aeruginosa and for PBP2 in E. coli. Doripenem is also considered to have increased antipseudomonal activity compared to imipenem. Taking into consideration the role of PBP in the mechanism of action of carbapenems, little is known about this processes in $A$. baumannii $[21,46]$.

\section{Microbial activity}

Carbapenems are beta-lactam antibiotics active against a wide range of Gram-positive and Gram-negative bacteria, including aerobes as well as anaerobes. However, in contrast, none of the carbapenems is clinically useful against Enterococcus faecium and Stenotrophomonas maltophilia. Generally, imipenem, panipenem and doripenem are slightly more active versus Gram-positive bacteria, while meropenem, biapenem, ertapenem, and doripenem are more potent against Gram-negative organisms. Taking into consideration carbapenem activity against $A$. bauman$n i i$, it has been shown that imipenem and doripenem are more potent than meropenem. Moreover, the MIC values of doripenem are lower than presented by imipenem and meropenem for $A$. baumannii. The studies concerning analysis of carbapenems $\mathrm{MIC}_{90}$ values among Acinetobacter spp., revealed more than eightfold higher $\mathrm{MIC}_{90}$ for ertapenem, comparing to other carbapenems [45-47].

\section{Clinical use}

As MDR (multidrug-resistant) pathogens emerge worldwide, in many cases carbapenems remain the "last-resort" antimicrobials used in empirical as well targeted therapy of severe infections. Furthermore, increasing phenomenon of carbapenem resistance limits available clinical options and stresses the need of development of combined therapy. Carbapenems can be combined with other antimicrobials to achieve an effective and safe therapy for serious $A$. baumannii infections [21, 46]. The possible advantages of combined therapy in comparison to monotherapy 
comprise a broader antibacterial spectrum, synergistic effects, and decreased risk for emerging resistance during therapy.

Many combinations have recently been reported in the scientific literature. In vitro study conducted by Fernández-Cuenca and co-workers revealed the additive effect of imipenem and azithromycin in the group of clinical strains of $A$. baumannii [49]. Moreover, Principe and co-authors demonstrated in vitro synergistic activity of tigecycline in combination with imipenem against tigecycline non-susceptible $A$. baumannii strains [50]. Another study concerning combination therapy showed synergistic antibacterial effect of imipenem with colistin among $100 \%$ of analysed imipenem- and meropenem-resistant $A$. baumannii isolates. Furthermore, the triple combinations of meropenem, sulbactam and colistin exhibited synergy against $96.7 \%$ of MDR A. baumannii [51]. Further studies concerning the combination of meropenem and ciprofloxacin revealed a small synergistic effect against the $A$. baumannii intensive care unit strains [52]. The research of Kiffer and co-workers showed that the combinations of meropenem and sulbactam may show synergism or partial synergism for analysed MDR A. baumannii isolates [53]. In the recent study, a combination of meropenem with minocycline was synergistic to tested XDR (extensively drug-resistant) A. baumannii isolates, but neither showed bactericidal activity alone. Furthermore, the authors observed that also colistin and meropenem presented synergistic effect and showed bactericidal activity against all tested strains [54, 55].

\section{Carbapenem resistance}

While carbapenems representing group 2 (meropenem, imipenem-cilastatin and doripenem) have been regarded as antimicrobials of "last-resort" in MDR A. baumannii infection therapy, recently a rapid increase in the rates of carbapenem resistant $A$. baumannii was observed [5]. Studies performed by National Healthcare Safety Network showed carbapenem resistance among $33 \%$ of $A$. baumannii strains derived from medical centres in the United States of America [14]. Moreover, Reddy and co-authors revealed the disturbing tendency of sharp increase in the rates of CRAb (carbapenem-resistant $A$. baumannii), from $1 \%$ in 2003 , to $58 \%$ in 2008 , associated with a more than twofold rise in occurrence of $A$. baumannii isolates [56]. A multinational study showed that $A$. baumannii imipenem susceptibility rates were higher for strains isolated from Europe and North America than those from Latin America and the Asia-Pacific Rim [57]. Another microbiological surveillance reports have

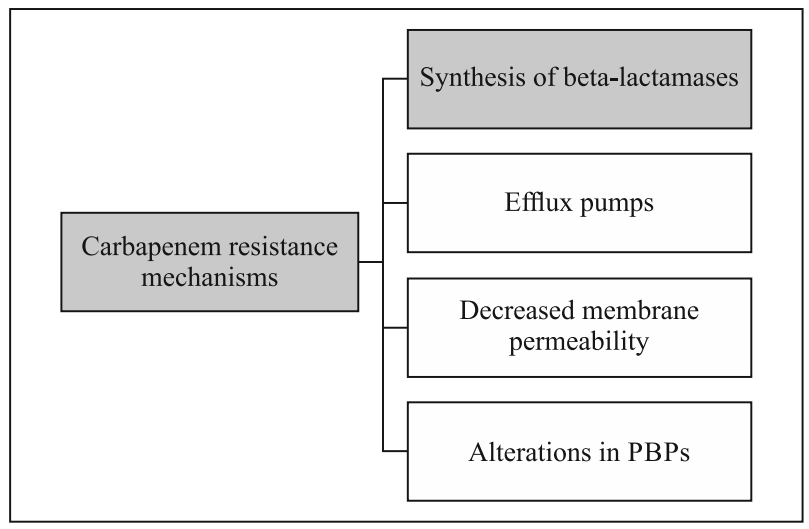

Figure 1. Mechanisms responsible for Acinetobacter baumannii carbapenem resistance. PBPs - penicillin-binding proteins.

revealed substantial rates of multidrug resistance in A. baumannii, which suggest that this bacterium became a growing public-health problem nowadays $[8,58]$. Growing prevalence of carbapenem-resistant $A$. baumannii isolates is considered as a threat to healthcare and patient safety worldwide, significantly reducing the ability to cure the infections. The progressive spread of CRAb strains is resulting in an urgent need for efficient detection, surveillance and guidance for infection prevention and control [58].

Mechanisms mediating $A$. baumannii resistance to carbapenems are presented in the Figure 1. The fusion of these mechanisms may result in high levels of carbapenem resistance in A. baumannii strains [46].

\section{Non-enzymatic mechanisms}

While carbapenems enter the bacterial cell via certain types of porins, their reduced expression play a role in the resistance to these antibiotics. The most characterised porin among $A$. baumannii is the carbapenem-associated OMP (CarO) [10]. According to Catel-Ferreira and co-workers, decrease in the expression of CarO results in the reduction of susceptibility to imipenem [59]. Another carbapenem-associated OMP is 33- to 36-kDa protein. Carbapenem resistance in the epidemic strain analysed by del Mar Tomás and co-workers was considered to be caused by the loss of 33- to 36-kDa OMP [60]. Efflux pumps extrude antimicrobials from the bacterial cells, which may result in resistance to a wide spectrum of antimicrobial agents as well as disinfectants. While five families of EP are associated with increased resistance of bacteria, only three of them are represented in A. baumannii: multidrug and toxic compound extrusion (MATE) family, the major facilitator superfamily (MFS), and 
the resistance-nodulation-cell division (RND) family. In $A$. baumannii the major efflux pump associated with carbapenem resistance is AdeABC. This EP belongs to RND family and is comprised of three-components: AdeA - membrane fusion protein, AdeB - inner membrane protein channel, and AdeC - outer membrane protein channel [61]. Overexpression of this EP is regulated by adeS and adeR genes contributing to increased resistance to antimicrobials, inter alia: meropenem, fluoroquinolones, tetracyclines, chloramphenicol as well as aminoglycosides [10]. Another mechanism responsible for carbapenem resistance in A. baumannii is related to alterations in penicillin-binding proteins. This mechanism confers resistance to beta-lactam antimicrobials in many bacterial species, however the data concerning this phenomenon in A. baumannii are limited. Although several PBP proteins have been described in $A$. baumannii so far, their role is attributed only to low-level carbapenem resistance [62, 63]. Furthermore, studies concerning CRAb strains with multiple mechanisms responsible for this process, revealed reduced expression of selected PBP proteins [63].

\section{Enzymatic mechanisms}

Bacterial enzymes responsible for hydrolytic inactivation of different groups of beta-lactam antibiotics, such as penicillins, cephalosporins, monobactams and carbapenems are represented by beta-lactamases. These enzymes are categorised according to the sequence homology into four molecular classes: A, $\mathrm{B}, \mathrm{C}$ and $\mathrm{D}$.

Carbapenem resistance can be mediated by hyperproduction or derepression of Ambler class $\mathrm{C}$ beta-lactamases (AmpC beta-lactamases), selected ESBL (extended-spectrum beta-lactamases) and carbapenemases [64]. In accordance with the current knowledge the most significant mechanism of carbapenem resistance in $A$. baumannii is associated with carbapenemases, the most versatile family of beta-lactamases. The Figure 2 shows the clinically relevant carbapenemases occurring among $A$. baumannii.

Based on the participation of divalent cations in enzyme activation, carbapenemases can be segregated into metallo-carbapenemases (class B) and non-metallo-carbapenemases (class A, C and D) [65]. Both groups of enzymes comprise the resistance to carbapenems by breaking the amide bond of the beta-lactam ring, however the mechanism of this process differs substantially. The hydrolysis of carbapenems mediated by metalloenzymes involves a water molecule, which is coordinated to a divalent cation (zinc) in order to activate and disrupt the beta-lactam ring. It is worth

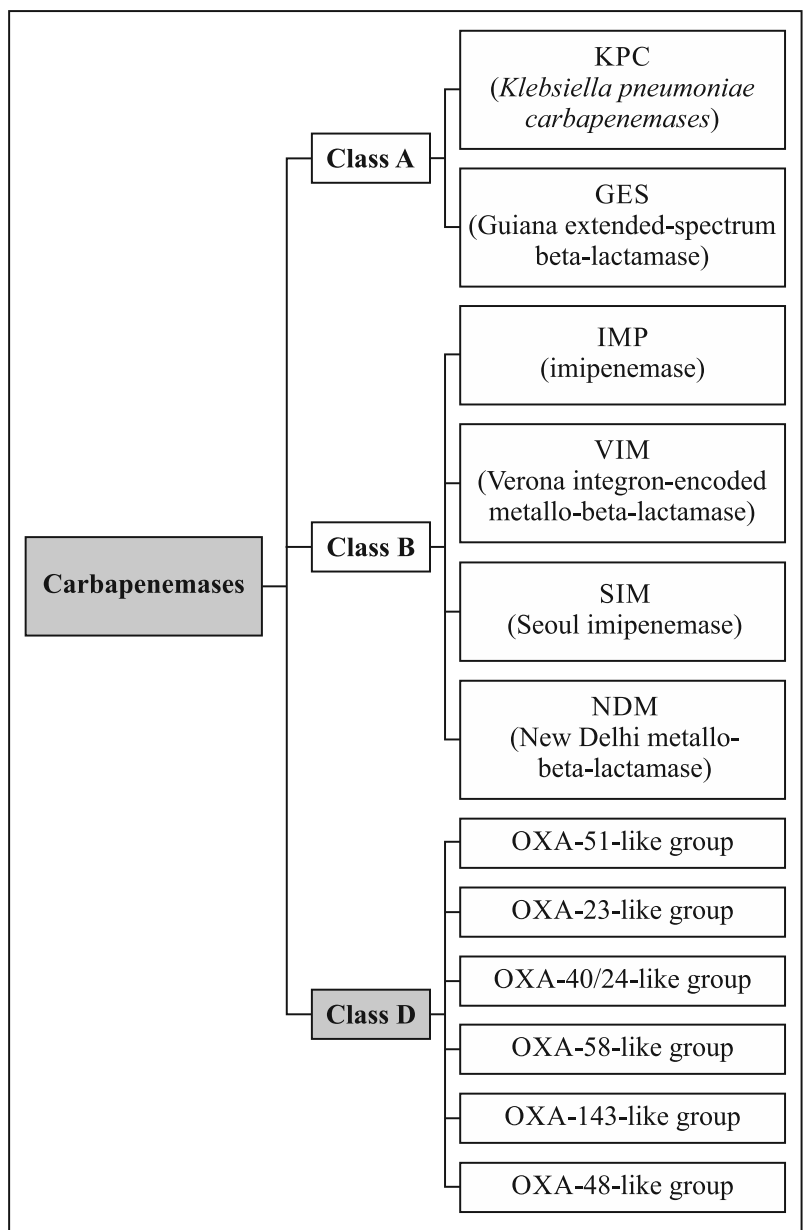

Figure 2. Clinically relevant carbapenemases occurring among Acinetobacter baumannii.

emphasizing that metallo-beta-lactamases do not form the covalent acyl-enzyme intermediate $[65,66]$.

The hydrolytic inactivation of carbapenem antibiotics performed by non-metallo-carbapenemases includes acylation and deacylation reactions. In the first step, a conserved serine located in the active site of enzymes, attacks the beta-lactam amide bond, assembling an acyl-enzyme complex. This intermediate is hydrolysed afterwards by a deacylating water molecule, forming the hydrolysed product that is released from the active site of the enzyme [65]. It is considered that carbapenem hydrolysing class $\mathrm{D}$ beta-lactamases (CHDL) are the most common factor of carbapenem resistance in $A$. baumannii strains. These enzymes are referred as OXAs (oxacillinases) due to their ability to hydrolyse isoxazolylpenicillin - oxacillin much faster than benzylpenicillin [67, 68]. Among A. baumannii there have been identified so far six groups of OXA carbapenemases represented by: OXA-51-like, OXA-23-like, OXA-40/24-like, OXA-58-like, OXA-143-like, and OXA-48-like [9, 69]. 
The groups, occurrence, structure, catalytic mechanism and inhibitors of $A$. baumannii carbapenemases have been thoroughly described in the Supplementary File 1. The acquired carbapenem-hydrolysing OXA-type beta-lactamases carried by Acinetobacter baumannii strains have been listed in Supplementary Table 1 and the metallo-beta-lactamases reported among $A$. baumannii strains - in Supplementary Table 2.

\section{Carbapenemase encoding genes: genetic location transfer and mechanisms of control}

Carbapenemase encoding genes can be carried within A. baumannii genome on chromosome and/ or plasmids. Furthermore, genetic determinants of carbapenemases are often associated with mobile genetic elements, such as insertion sequences (IS), integrons, transposons, resistance islands (RI), and plasmids, often contributing to their acquisition, dissemination and regulation among bacterial isolates [21].

\section{Insertion sequences}

Insertion sequences, also described as insertion sequence elements are DNA segments not exceeding in size $2500 \mathrm{bp}$, and therefore considered to be the smallest mobile DNA elements. IS can contribute to resistance by: providing additional promoters in order to amplify the expression of certain genes, disarranging the coding sequences of particular genes, and allowing the dissemination of gene cassettes among bacterial strains [21]. According to the Reference Centre for Bacterial Insertion Sequences (ISfinder), 30 different types of IS were described in A. baumannii so far [70]. The insertion sequence of particular importance and prevalence is represented by IS $A b a 1$. It is considered that the first report of $A$ baumannii IS $A b a 1$ concerned the isolate from 2001, however it turned out that the sequences corresponding to this element had been reported previously. While the presence of ISAbal has been found in association with numerous antimicrobial resistance determinants, its role in mobilization of OXA-type carbapenemases has been reported in particular [69]. The association of ISAbal and OXA-type carbapenemases encoding genes includes intrinsic as well as acquired enzymes. Turton and co-authors suggested that the location of ISAbal sequence upstream of blaOXA-51-like is providing the gene promoter that may result in resistance to carbapenems [71]. The ISAbal has been also found in association with $b l a_{\text {OXA-23-like }}$ and $b l a_{\text {OXA-58-like }}$ genes [71-73]. Nevertheless, several authors have suggested that overexpression of these CHDLs may be also associated with the presence of ISAbal flanking these genes, and therefore may result with decreased susceptibility to carbapenems $[72,74,75]$.

\section{Integrons}

Integrons are genetic elements that are capable of capturing antibiotic resistance determinants and able to promote their transcription and expression [76]. In recent decades it has turned out that integrons play a vital role in the acquisition and dissemination of antibiotic resistance genes, particularly among Gram-negative bacteria [77]. Integrons can be classified into several classes according to the relative homology of integrase encoding gene (intI) [77]. It is considered that the occurrence of integrons among A. baumannii strains may suggest their high epidemic potential. More than five classes of integrons have been described so far, with the class 1 integrons to be the most prevalent among multidrug-resistant A. baumannii clinical strains worldwide [78-80]. Moreover, it has been noted so far that among A. baumannii isolates class 1 integrons can carry only metallo-beta-lactamases, neither OXA nor KPC carbapenemase encoding genes. An example of imipenem-resistant $A$. baumannii strain carrying $b l a_{\mathrm{IMP}-5}$ gene within class 1 integron was reported by Da Silva and co-workers [81]. Also the studies of Huang and co-authors revealed the presence of multidrug-resistant $A$. baumannii isolates carrying within the class 1 integron the $b l a_{\mathrm{VIM}-11}$ gene [82]. Additionally, the occurrence of $b l a_{\text {SIM-1 }}$ was detected in class 1 integrons among seven clinical isolates of $A$. baumannii from Korea [83]. While the genetic determinants of IMP, VIM and SIM-type enzymes were found on class 1 integrons of $A$. baumannii, bla $_{\mathrm{NDM}}$ have not been found till date [84].

\section{Resistance islands}

Bacterial resistance islands are defined as a particular region within the genome harbouring a high concentration of horizontally transferred antimicrobial resistance genes [9,21]. There have been numerous RI described among $A$. baumannii strains so far, represented by AbaR1, AbaR3, AbaR4, AbaR5-Aba19, AbaR25, and others [85-87]. The first $A$. baumannii resistance island (AbaR1) was reported in multidrug-resistant AYE strain, in 2006 by Fournier and co-workers [88]. The whole-genome sequencing of the strain revealed the presence of $86 \mathrm{~kb}$ resistance island, which harboured a cluster of 45 resistance genes, including $b l a_{\text {OXA-69 }}$ (member of bla $a_{\text {OXA-51-like }}$ group) [88]. Studies concerning the group of carbapenem-resistant A. baumannii isolates in Latvia disclosed the prevalence of AbaR25 resistance island. The Acinetobacter 
baumannii strain carrying the AbaR25 (variant of AbaR4) was linked to international clone II/ST2 and carried within the resistant island the $b l a_{\text {OXA-23-like }}$ carbapenemase gene [87]. Furthermore, studies concerning CRAb isolates from Asia revealed the presence of two AbaR4-type resistance islands (D36 and $\mathrm{AB} 210$ ) that carried $b a_{\text {OXA-23-like }}$ genes [89].

\section{Plasmids}

Plasmids are circular or linear double-stranded DNA molecules distinct from chromosomal DNA, often transferred by means of conjugation [90, 91]. Studies concerning the sequence analysis of $A$. baumannii plasmid replicons revealed the differences comparing to other prokaryotic species, suggesting that $A$. baumannii strains may carry distinct set of plasmid types $[92,93]$. It is believed that the most clinically relevant carbapenemases are often associated with plasmids [68]. While $A$. baumannii plasmids may vary in size as well as genetic content, Bertini and co-authors proposed their classification based on the replicase gene sequences [92]. Towner and co-authors analysing the group of $96 \mathrm{MDR}$ A. baumannii clinical isolates from 17 European countries, confirmed the presence at least 1 (with a maximum of 4 ) out of 19 replicases (rep) gene groups (GR) among all clinical strains tested. The largest group of strains belonged to GR6 (repAci6; 93 isolates), and the variations in rep gene content were even presented among epidemiologically related isolates. Further analysis of co-occurrence of rep genes and CHDL encoding genes revealed the association of $b l a_{\text {OXA-58-ike }}$ genes (22 strains) with repAci1, repAci3, repAci4, and repAciX genes, while bla $a_{\text {OXA-40-like }}$ (6 strains) were related to repAci2 and p2ABSDF0001, and bla $a_{\text {OXA-23-like }}$ (8 isolates) were correlated with repAci1 [93].

\section{Methods of detection of carbapenemases}

The clinical relevance of rapid detection of carbapenemases among $A$. baumannii strains is crucial due to selection of appropriate antibiotic therapy as well as prevention of the development of the outbreaks. Till date, there have been introduced a number of methods, differing in principles, accuracy and time of detection.

\section{Phenotypic methods}

Among phenotypic tests used in the detection of carbapenemases, methods based on the inhibition of the enzyme activity by specified inhibitors deserve particular attention. The culture-based techniques of metallo-beta-lactamases detection are, inter alia represented by double-disk synergy test and Etest
MBL (bioMeriéux, France). These methods, enabling to detect MBL carbapenemases in routine microbiology practice, utilize EDTA as the enzymes inhibitor [94, 95]. Another culture-based method, used for detection of carbapenemases among $A$. baumannii is represented by KPC combined disk assay. This technique utilizes boronic acid compound (BAC) as KPC inhibitor [96, 97].

\section{Biochemical methods}

Very recently, a novel and promising technique for the rapid and accurate detection of carbapenemases has been developed. The Carba NP test uses isolated bacterial colonies and is based on in vitro hydrolysis of imipenem. The carbapenemase activity is detected by colour change of $\mathrm{pH}$ indicator resulting from the hydrolysis of imipenem into a carboxylic derivative, leading to a decrease of the $\mathrm{pH}$ value [98-100]. While the application of Carba NP test in carbapenemase detection among Acinetobacter spp. strains encountered certain difficulties, a modified version of the assay the CarbAcineto NP test, has been developed. Dortet and co-authors performed the analysis of specificity and sensitivity of the CarbAcineto NP test and revealed $100 \%$ and $94.7 \%$, respectively [101]. Another method based on hydrolysis of carbapenem beta-lactam ring is Rapid CARB Blue Kit (Rosco Diagnostica, Taastrup, Denmark). The assay uses Tienam (commercially available imipenem) (Merck Sharp \& Dohme, France) as a substrate for carbapenemases and bromothymol blue as a pH indicator. The Rapid CARB Blue Kit has been developed for detection of carbapenemase among Acinetobacter spp., Enterobacteriaceae and Pseudomonas spp. strains [102, 103].

An additional approach to carbapenemase detection is associated with application of the ultraviolet (UV) spectrophotometry. The assay is performed for overnight cultures of $A$. baumannii which are centrifuged and sonicated, and then followed by UV spectrophotometric measurement (wavelength value of $297 \mathrm{~nm}$ for imipenem) of specific activity of carbapenemases. While this technique is considered to be efficient in detection of VIM, IMP and SIM carbapenemase producers, it is regarded to be unsuitable for testing for CHDL and NDM carbapenemases [104].

Another promising method of carbapenemase detection is based on the MALDI-TOF MS (matrix-assisted laser desorption/ionization time of flight mass spectrometry). According to the definition presented by Wieser and co-authors the method is based on the ionization of cocrystalized sample material by short laser pulses, and then so formed ions are accelerated and their time of flight is measured in a vacuum flight tube [105]. The application of MAL- 
DI-TOF MS in the detection of carbapenemases is based on the identification of native imipenem and its natural metabolite, both molecules are identified according to their different masses. In the studies by Kempf and co-workers the carbapenemase detection by MALDI-TOF MS method was carried among $106 \mathrm{~A}$. baumannii clinical isolates from France and Algeria. According to the authors, the method revealed a sensitivity and specificity of $100 \%$. While this technique is considered to be rapid and effective, it requires particularly expensive equipment and specialized laboratory personnel [106].

\section{Molecular methods}

Among techniques used in the detection of carbapenemases, nucleic acid based methods deserve particular attention. These methods are considered to be the "gold standard" characterised with high specificity and sensitivity. Currently, the most of the molecular techniques used in the detection of carbapenemases are based on the polymerase chain reaction (PCR). A single end-point PCR is one of the first molecular methods used in epidemiological as well as resistance studies [107].

While single end-point PCR allows detection of only one gene (per reaction), the multiplex PCR technique enables identification of different target genes in one reaction. Several multiplex PCR assays detecting carbapenemases in $A$. baumannii strains were described so far. Woodford and co-authors developed the multiplex PCR technique which allows identification and distinguishing of alleles encoding four groups of OXA carbapenemases - OXA-23-like, OXA-40/24-like, OXA-58-like, and OXA-51-like among Acinetobacter spp. [108]. Another reliable and rapid method used in the detection of carbapenemases was developed by Poirel and co-workers. The authors defined and evaluated the technique that allows detecting 11 carbapenemase encoding genes $\left(b l a_{\text {IMP }}\right.$, bla $a_{\mathrm{VIM}}, b l a_{\mathrm{NDM}}, b l a_{\mathrm{SPM}}, b l a_{\mathrm{AIM}}, b l a_{\mathrm{DIM}}$, bla $_{\mathrm{GIM}}$, bla ${ }_{\mathrm{SIM}}$, $b l a_{\mathrm{KPC}}, b l a_{\mathrm{BIC}}$, and $\left.b l a_{\mathrm{OXA}-48}\right)$ in three multiplex PCR reactions. Application of the optimized conditions in each reaction mixture allowed obtaining distinct PCR amplicons for respective carbapenemase genes [109].

Another step forward in the molecular diagnostics was the introduction of real-time PCR (also known as quantitative PCR). While the end-point PCR requires gel electrophoresis in amplicon detection, the real-time PCR technique utilizes amplification and detection in a single step. The product detection can be obtained according to two main approaches: applying a dye that binds to double-stranded DNA (e.g. SYBR Green, EvaGreen) or implementing sequence specific probes (e.g. TaqMan ${ }^{\circledR}$, Molecular Beacons,
Scorpion) [107, 110]. Furthermore, real-time PCR in comparison to end-point PCR method allows to obtain a quantitative result and also to perform the reaction in a shorter period of time. An example of the application of real-time PCR in carbapenemase detection among $A$ baumannii isolates was reported by Pasanen and co-workers. The authors described the multiplex real-time PCR assay that detected carbapenemase genes for KPC, VIM, IMP, GES-1/-10, OXA-48, NDM, GIM-1, SPM-1, IMI/NMC-A, SME, CMY-10, SFC-1, SIM-1, OXA-23-like, OXA-40/24-like, OXA-58, and ISAba1-OXA-51-like in just two separate reactions. This SYBR Green based assay demonstrated the good performance detecting relevant carbapenemases among clinical isolates of $A$. baumannii [111]. Despite the SYBR Green chemistry based real-time PCR methods, sequence specific TaqMan ${ }^{\circledast}$ probes are applied in carbapenemase detection among $A$. baumannii strains. Huang and co-workers described the multiplex TaqMan ${ }^{\circledR}$ real-time PCR assay for simultaneous detection of four carbapenem-resistance genes - bla $a_{\text {OXA-23-like, }}$ $b l a_{\text {OXA-51-like }}, b l a_{\text {OXA-40/24-like }}$, and $b l a_{\text {OXA-58-like }}$. The assay demonstrated a high specificity, suggesting its application in early diagnosis of carbapenem resistant A. baumannii [112].

Another method of gene amplification is represented by loop-mediated isothermal amplification assay (LAMP). This technique is based on autocycling strand displacement DNA synthesis performed under isothermal conditions in the presence of Bst DNA polymerase. Solanki and co-workers evaluated the application of LAMP method in the detection of $b l a_{\mathrm{NDM}-1}$ and $b l a_{\mathrm{KPC}}$ genes among carbapenem resistant Gram-negative isolates, including $A$. baumannii. The authors compared the LAMP assay with phenotypic and PCR based methods. The studies revealed that LAMP technique appeared to be more sensitive than conventional PCR, indicating the presence of $b l a_{\mathrm{NDM}-1}$ and $b l a_{\mathrm{KPC}}$ genes among four strains that were not detected by PCR. The authors also concluded that LAMP method with its high sensitivity and short turnaround time could be considered as a rapid and accurate point-of-care assay in the detection of NDM and KPC carbapenemases [113, 114].

Yet another latest molecular technique is the DNA microarray, also known as DNA chip or bio-chip. This method is based on hybridization of nucleic acid sample to a large set of oligonucleotide probes, which are attached to a solid surface [115]. DNA microarray allows for the simultaneous analysis of multiple genes; therefore, it could be applied in the identification of various resistance as well as virulence genes among tested strains within one assay. Dally and co-work- 
Table 3. Selected methods used in the detection of carbapenemases in Acinetobacter baumannii

\begin{tabular}{|l|c|c|}
\hline Group & Method & References \\
\hline Phenotypic & Double-disk synergy test & {$[94]$} \\
& Disk combination method & {$[97]$} \\
& Etest MBL & {$[95]$} \\
\hline \multirow{2}{*}{ Biochemical } & CarbAcineto NP test & {$[101]$} \\
& Rapid CARB Blue Kit & {$[102]$} \\
& UV spectrophotometry & {$[104]$} \\
& MALDI-TOF MS & {$[106]$} \\
\hline \multirow{2}{*}{ Molecular } & PCR & {$[108,109]$} \\
& Real-time PCR & {$[111,112,117]$} \\
& LAMP & {$[113,114]$} \\
& DNA chip & {$[116,118,119]$} \\
\hline
\end{tabular}

ers developed a microarray which allows detecting 91 target sequences of resistance determinants among multidrug-resistant $A$. baumannii strains within 4 hours from bacterial culture to the result. Among resistance determinants included in the study, carbapenemase genes were represented by: bla ${ }_{\mathrm{OXA}-23}$, $b l a_{\text {OXA- }-37}, b l a_{\text {OXA-40, }}, b l a_{\text {OXA-48, }}, b l a_{\text {OXA-51 }}, b l a_{\text {OXA-58, }}, b l a_{\mathrm{KPC}}$, $b l a_{\mathrm{VIM}}, b l a_{\mathrm{IMP}-1}, b l a_{\mathrm{IMP}-2}, b l a_{\mathrm{SIM}}, b l a_{\mathrm{GES}}$, and bla $_{\mathrm{NDM}}$. In the group of $60 \mathrm{~A}$. baumannii strains the carbapenemase genes were detected among: 14, 13, 11, and 8 strains, carrying $b l a_{\text {OXA-23-like, }}, b l a_{\text {OXA-40/24-like }}, b l a_{\text {OXA-58-like, }}$, and MBL genes, respectively. Furthermore, 10 isolates harboured deregulated $b l a_{\text {OXA-51-like }}$ genes due to ISAbal integration. Application of the microarray assay in the detection of carbapenemases revealed complete concordance with singleplex PCR provided by German National Reference Centre for Gram-negative Pathogens. Taking into consideration microarray's reliability and short handling time, it can be applied as a fast and reliable tool in resistance studies concerning both adequate treatment selection of intensive therapy patients as well as epidemiological studies [116]. The overview of mentioned above methods is presented in Table 3 .

\section{Conclusions}

Over the last decades $A$. baumannii has emerged as an important nosocomial pathogen. While carbapenems have long been considered as an effective antimicrobial against $A$. baumannii infections, emerging resistance has been causing substantial difficulties in the treatment of this nosocomial pathogen worldwide. Currently, the most relevant mechanism of A. baumannii carbapenem resistance is associated with the beta-lactams hydrolysing enzymes represented by OXA-type and MBL carbapenemases. While laboratory detection and identification of carbapenemases among $A$. baumannii is troublesome, numerous promising methods have been described. The techniques which deserve particular attention belong to unsophisticated screening methods that can be used in routine microbiological laboratory, modern methods based on mass spectroscopy, and molecular biology, which enable detection of different carbapenemases in one run. While carbapenemase-producing A. baumannii strains are often multidrug-resistant, studies concerning carbapenemases inhibitors may offer a new insight into effective treatment of MDR A. baumannii.

\section{References}

1. Vidal R, Dominguez M, Urrutia $\mathrm{H}$ et al. Biofilm formation by Acinetobacter baumannii. Microbios. 1996;86:49-58. PMID: 8771775.

2. Lee HW, Koh YM, Kim J et al. Capacity of multidrug-resistant clinical isolates of Acinetobacter baumannii to form biofilm and adhere to epithelial cell surfaces. Clin Microbiol Infect. 2008;14:49-54. doi: 10.1111/j.1469-0691.2007.01842.x.

3. Garnacho-Montero J, Amaya-Villar R. Multiresistant Acinetobacter baumannii infections: epidemiology and management. Curr Opin Infect Dis. 2010;23:332-339. doi: 10.1097/ QCO.0b013e32833ae38b.

4. Cerqueira GM, Peleg AY. Insights into Acinetobacter baumannii pathogenicity. IUBMB Life. 2011;63:1055-1060. doi: 10.1002/iub.533.

5. Pogue JM, Mann T, Barber KE, Kaye KS. Carbapenem-resistant Acinetobacter baumannii: epidemiology, surveillance and management. Expert Rev Anti Infect Ther. 2013;11:383-393. doi: 10.1586/eri.13.14.

6. McConnell MJ, Actis L, Pachón J. Acinetobacter baumannii: human infections, factors contributing to pathogenesis and animal models. FEMS Microbiol Rev. 2013;37:130-155. doi: 10.1111/j.1574-6976.2012.00344.x.

7. Antunes LC, Visca P, Towner KJ. Acinetobacter baumannii: evolution of a global pathogen. Pathog Dis. 2014;71:292-301. doi: 10.1111/2049-632X.12125.

8. Karageorgopoulos DE, Falagas ME. Current control and treatment of multidrug-resistant Acinetobacter baumannii infections. Lancet Infect Dis. 2008;8:751-762. doi: 10.1016/ S1473-3099(08)70279-2.

9. Evans BA, Hamouda A, Amyes SG. The rise of carbapenem-resistant Acinetobacter baumannii. Curr Pharm Des. 2013;19:223-238. PMID: 22894617.

10. Abbott I, Cerqueira GM, Bhuiyan S, Peleg AY. Carbapenem resistance in Acinetobacter baumannii: laboratory challenges, mechanistic insights and therapeutic strategies. Expert Rev Anti Infect Ther. 2013;11:395-409. doi: 10.1586/eri.13.21.

11. Dijkshoorn L, Nemec A, Seifert H. An increasing threat in hospitals: multidrug-resistant Acinetobacter baumannii. Nat Rev Microbiol. 2007;5:939-951. doi: 10.1038/nrmicro1789.

12. Zhang HZ, Zhang JS, Qiao L. The Acinetobacter baumannii group: a systemic review. World J Emerg Med. 2013;4:169-174. doi: 10.5847/wjem.j.1920-8642.2013.03.002.

13. Lemos EV, de la Hoz FP, Einarson TR et al. Carbapenem resistance and mortality in patients with Acinetobacter baumannii infection: systematic review and meta-analysis. Clin Microbiol Infect. 2014;20:416-423. doi: 10.1111/14690691.12363.

14. Hidron AI, Edwards JR, Patel J et al. NHSN annual update: antimicrobial-resistant pathogens associated with healthca- 
re-associated infections: annual summary of data reported to the National Healthcare Safety Network at the Centers for Disease Control and Prevention, 2006-2007. Infect. Control Hosp. Epidemiol. 2008;29:996-1011. doi: 10.1086/591861.

15. Falagas ME, Karveli EA, Kelesidis I, Kelesidis T. Community -acquired Acinetobacter infections. Eur J Clin Microbiol Infect Dis. 2007;2612:857-868. doi: 10.1007/s10096-007-0365-6.

16. Dexter C, Murray GL, Paulsen IT, Peleg AY. Community -acquired Acinetobacter baumannii: clinical characteristics, epidemiology and pathogenesis. Expert Rev Anti Infect Ther. 2015;13:567-573. doi: 10.1586/14787210.2015.1025055.

17. Joly-Guillou ML. Clinical impact and pathogenicity of Acinetobacter. Clin Microbiol Infect. 2005;11:868-873. doi: 10.1111/j.1469-0691.2005.01227.x.

18. Teicher CL, Ronat JB, Fakhri RM et al. Antimicrobial drug-resistant bacteria isolated from Syrian war-injured patients, August 2011-March 2013. Emerg Infect Dis. 2014;20:1949-1951. doi: 10.3201/eid2011.140835.

19. Oncül O, Keskin O, Acar HV et al. Hospital-acquired infections following the 1999 Marmara earthquake.J Hosp Infect. 2002;51:47-51. doi: 10.1053/jhin.2002.1205.

20. Uçkay I, Sax H, Harbarth S, Bernard L, Pittet D. Multi-resistant infections in repatriated patients after natural disasters: lessons learned from the 2004 tsunami for hospital infection control. J Hosp Infect. 2008;68:1-8. doi: 10.1016/j. jhin.2007.10.018.

21. Roca I, Espinal P, Vila-Farrés X, Vila J. The Acinetobacter baumannii Oxymoron: Commensal Hospital Dweller Turned Pan-Drug-Resistant Menace. Front Microbiol. 2012;3:148. doi: 10.3389/fmicb.2012.00148.

22. Luke NR, Sauberan SL, Russo TA et al. Identification and characterization of a glycosyltransferase involved in Acinetobacter baumannii lipopolysaccharide core biosynthesis. Infect Immun. 2010;78:2017-2023. doi: 10.1128/IAI.00016-10.

23. Knapp S, Wieland CW, Florquin S et al. Differential roles of CD14 and toll-like receptors 4 and 2 in murine Acinetobacter pneumonia. Am J Respir Crit Care Med. 2006;173:122-129. doi: 10.1164/rccm.200505-730OC.

24. Kim CH, Jeong YJ, Lee $\mathrm{J}$ et al. Essential role of toll-like receptor 4 in Acinetobacter baumannii-induced immune responses in immune cells. Microb Pathog. 2013;54:20-25. doi: 10.1016/j.micpath.2012.08.008.

25. Erridge C, Moncayo-Nieto OL, Morgan R, Young M, Poxton IR. Acinetobacter baumannii lipopolysaccharides are potent stimulators of human monocyte activation via Toll-like receptor 4 signalling. J Med Microbiol. 2007;56:165-171. doi: 10.1099/jmm.0.46823-0.

26. Russo TA, Luke NR, Beanan JM et al. The K1 capsular polysaccharide of Acinetobacter baumannii strain 307-0294 is a major virulence factor. Infect Immun. 2010;78:3993-4000. doi: 10.1128/IAI.00366-10.

27. Choi CH, Lee EY, Lee YC et al. Outer membrane protein 38 of Acinetobacter baumannii localizes to the mitochondria and induces apoptosis of epithelial cells. Cell Microbiol. 2005;7:1127-1138. doi: 10.1111/j.1462-5822.2005.00538.x.

28. Choi CH, Lee JS, Lee YC, Park TI, Lee JC.Acinetobacter baumannii invades epithelial cells and outer membrane protein A mediates interactions with epithelial cells. BMC Microbiol. 2008;8:216-227. doi: 10.1186/1471-2180-8-216.

29. Ellis TN, Kuehn MJ. Virulence and immunomodulatory roles of bacterial outer membrane vesicles. Microbiol Mol Biol Rev. 2010;74:81-94. doi: 10.1128/MMBR.00031-09.

30. Kwon SO, Gho YS, Lee JC, Kim SI. Proteome analysis of outer membrane vesicles from a clinical Acinetobacter bau- mannii isolate. FEMS Microbiol Lett. 2009;297:150-156. doi: 10.1111/j.1574-6968.2009.01669.x.

31. Jun SH, Lee JH, Kim BR et al. Acinetobacter baumannii outer membrane vesicles elicit a potent innate immune response via membrane proteins. PLoS One. 2013;8:e71751. doi: 10.1371/ journal.pone.0071751.

32. Jacobs AC, Hood I, Boyd KL et al. Inactivation of phospholipase D diminishes Acinetobacter baumannii pathogenesis. Infect Immun. 2010;78:1952-1962. doi: 10.1128/IAI.00889-09.

33. Donlan RM, Costerton JW. Biofilms: survival mechanisms of clinically relevant microorganisms. Clin Microbiol Rev. 2002;15:167-193. doi: 10.1128/CMR.15.2.167-193.2002.

34. Villegas MV, Hartstein AI. Acinetobacter outbreaks, 1977-2000. Infect Control Hosp Epidemiol. 2003;24:284-295. doi: $10.1086 / 502205$.

35. Rodríguez-Baño J, Martí S, Soto S et al. Biofilm formation in Acinetobacter baumannii: associated features and clinical implications. Clin Microbiol Infect. 2008;14:276-278. doi: 10.1111/j.1469-0691.2007.01916.x.

36. Djeribi R, Bouchloukh W, Jouenne T, Menaa B. Characterization of bacterial biofilms formed on urinary catheters. $\mathrm{Am}$ JInfect Control. 2012;40:854-859. doi: 10.1016/j.ajic.2011.10.009.

37. Badave GK, Kulkarni D. Biofilm Producing Multidrug Resistant Acinetobacter baumannii: An Emerging Challenge. J Clin Diagn Res. 2015;9:DC08-DC10. doi: 10.7860/ JCDR/2015/11014.5398.

38. Tomaras AP, Dorsey CW, Edelmann RE, Actis LA. Attachment to and biofilm formation on abiotic surfaces by Acinetobacter baumannii: involvement of a novel chaperone-usher pili assembly system. Microbiology. 2003;149:3473-3484. doi: 10.1099/mic.0.26541-0.

39. Tomaras AP, Flagler MJ, Dorsey CW, Gaddy JA, Actis LA. Characterization of a two-component regulatory system from Acinetobacter baumannii that controls biofilm formation and cellular morphology. Microbiology. 2008;154:3398-3409. doi: 10.1099/mic.0.2008/019471-0.

40. Loehfelm TW, Luke NR, Campagnari AA. Identification and characterization of an Acinetobacter baumannii biofilm-associated protein. J Bacteriol. 2008;190:1036-1044. doi: 10.1128/ JB.01416-07.

41. Choi AH, Slamti L, Avci FY, Pier GB, Maira-Litrán T. The pgaABCD locus of Acinetobacter baumannii encodes the production of poly-beta-1-6- $N$-acetylglucosamine, which is critical for biofilm formation. J Bacteriol. 2009;191:5953-5963. doi: 10.1128/JB.00647-09.

42. Gaddy JA, Actis LA. Regulation of Acinetobacter baumannii biofilm formation. Future Microbiol. 2009;4:273-278. doi: 10.2217/fmb.09.5.

43. Niu C, Clemmer KM, Bonomo RA, Rather PN. Isolation and characterization of an autoinducer synthase from Acinetobacter baumannii. J Bacteriol. 2008;190:3386-3392. doi: 10.1128/JB.01929-07.

44. Leclercq R, Cantón R, Brown DF et al. EUCAST expert rules in antimicrobial susceptibility testing. Clin Microbiol Infect. 2013;19:141-160. doi: 10.1111/j.1469-0691.2011.03703.x.

45. Zhanel GG, Wiebe R, Dilay L et al. Comparative review of the carbapenems. Drugs. 2007;67:1027-1052. doi: 00126667/07/0007-1027/\$49.95/0.

46. Papp-Wallace KM, Endimiani A, Taracila MA, Bonomo RA. Carbapenems: past, present, and future. Antimicrob Agents Chemother. 2011;55:4943-4960. doi: 10.1128/AAC.00296-11.

47. Zhanel GG, Johanson C, Embil JM et al. Ertapenem: review of a new carbapenem. Expert Rev Anti Infect Ther. 2005;3:23-39. doi: 10.1586/14787210.3.1.23. 
48. Williamson R, Collatz E, Gutmann L. Mechanisms of action of beta-lactam antibiotics and mechanisms of non-enzymatic resistance. Presse Med. 1986;20:2282-2289. PMID: 2949269.

49. Fernández-Cuenca F, Martínez-Martínez L, Pascual A, Perea EJ. In vitro activity of azithromycin in combination with amikacin, ceftazidime, ciprofloxacin or imipenem against clinical isolates of Acinobacter baumannii. Chemotherapy. 2003;49:24-26. doi: 10.1159/000069774.

50. Principe L, D’Arezzo S, Capone A, Petrosillo N, Visca P. In vitro activity of tigecycline in combination with various antimicrobials against multidrug resistant Acinetobacter baumannii. Ann Clin Microbiol Antimicrob. 2009;21:18. doi: 10.1186/1476-0711-8-18.

51. Pongpech P, Amornnopparattanakul S, Panapakdee S et al. Antibacterial activity of carbapenem-based combinations againts multidrug-resistant Acinetobacter baumannii. J Med Assoc Thai. 2010;93:161-171. PMID: 20301995.

52. Ermertcan S, Hoşgör M, Tünger O, Coşar G. Investigation of synergism of meropenem and ciprofloxacin against Pseudomonas aeruginosa and Acinetobacter strains isolated from intensive care unit infections. Scand J Infect Dis. 2001;33:818-821. PMID: 11760161.

53. Kiffer CR, Sampaio JL, Sinto $\mathrm{S}$ et al. In vitro synergy test of meropenem and sulbactam against clinical isolates of Acinetobacter baumannii. Diagn Microbiol Infect Dis. 2005;52:317-322. doi: 10.1016/j.diagmicrobio.2005.03.003.

54. Liang W, Liu XF, Huang J, Zhu DM, Li J, Zhang J. Activities of colistin- and minocycline-based combinations against extensive drug resistant Acinetobacter baumannii isolates from intensive care unit patients. BMC Infect Dis. 2011;27:109. doi: 10.1186/1471-2334-11-109.

55. Tängdén T. Combination antibiotic therapy for multidrug-resistant Gram-negative bacteria. Ups J Med Sci. 2014;119:149_ -153. doi: 10.3109/03009734.2014.899279.

56. Reddy T, Chopra T, Marchaim D et al. Trends in antimicrobial resistance of Acinetobacter baumannii isolates from a metropolitan Detroit health system. Antimicrob Agents Chemother. 2010;54:2235-2238. doi: 10.1128/AAC.01665-09.

57. Reinert RR, Low DE, Rossi F et al. Antimicrobial susceptibility among organisms from the Asia/Pacific Rim, Europe and Latin and North America collected as part of TEST and the in vitro activity of tigecycline. J Antimicrob Chemother. 2007;60:1018-1029. doi: 10.1093/jac/dkm310.

58. Carbapenemase-producing bacteria in Europe: interim results from the European Survey on carbapenemase-producing Enterobacteriaceae (EuSCAPE) project 2013. European Centre for Disease Prevention and Control Web site. 2013. Available at: http://www.ecdc.europa.eu. Accessed September 15, 2014.

59. Catel-Ferreira M, Coadou G, Molle V et al. Structure-function relationships of $\mathrm{CarO}$, the carbapenem resistance-associated outer membrane protein of Acinetobacter baumannii. Antimicrob Chemother. 2011;66:2053-2056. doi: 10.1093/jac/dkr267.

60. del Mar Tomás M, Beceiro A, Pérez A et al. Cloning and functional analysis of the gene encoding the 33- to 36-kilodalton outer membrane protein associated with carbapenem resistance in Acinetobacter baumannii. Antimicrob Agents Chemother. 2005;49:5172-5175. doi: 10.1128/ AAC.49.12.5172-5175.2005.

61. Wieczorek P, Sacha P, Hauschild T, Zórawski M, Krawczyk M, Tryniszewska E. Multidrug resistant Acinetobacter baumannii - the role of AdeABC (RND family) efflux pump in resistance to antibiotics. Folia Histochem Cytobiol. 2008;46:257-267. doi: 10.2478/v10042-008-0056-x.

62. Gehrlein M, Leying H, Cullmann W, Wendt S, Opferkuch W. Imipenem resistance in Acinetobacter baumanii is due to altered penicillin-binding proteins. Chemotherapy. 1991;37:405-412. PMID: 1760939.

63. Fernández-Cuenca F, Martínez-Martínez L, Conejo MC, Ayala JA, Perea EJ, Pascual A. Relationship between beta-lactamase production, outer membrane protein and penicillin-binding protein profiles on the activity of carbapenems against clinical isolates of Acinetobacter baumannii.J Antimicrob Chemother. 2003;51:565-574. doi: 10.1093/jac/dkg097.

64. Patel G, Bonomo RA. "Stormy waters ahead": global emergence of carbapenemases. Front Microbiol. 2013;14:48. doi: 10.3389/fmicb.2013.00048

65. Jeon JH, Lee JH, Lee JJ et al. Structural basis for carbapenem-hydrolyzing mechanisms of carbapenemases conferring antibiotic resistance. Int J Mol Sci. 2015;29:9654-9692. doi: 10.3390/ijms16059654.

66. Walsh TR, Toleman MA, Poirel L, Nordmann P. Metallo-beta-lactamases: the quiet before the storm? Clin Microbiol Rev. 2005;18:306-325 doi: 10.1128/CMR.18.2.306-325.2005.

67. Poirel L, Naas T, Nordmann P. Diversity, epidemiology, and genetics of class D beta-lactamases. Antimicrob Agents Chemother. 2010;54:24-38. doi: 10.1128/AAC.01512-08.

68. Diene SM, Rolain JM. Carbapenemase genes and genetic platforms in Gram-negative bacilli: Enterobacteriaceae, Pseudomonas and Acinetobacter species. Clin Microbiol Infect. 2014;20:831-838. doi: 10.1111/1469-0691.12655.

69. Evans BA, Amyes SG. OXA $\beta$-lactamases. Clin Microbiol Rev. 2014;27:241-263. doi: 10.1128/CMR.00117-13.

70. Siguier P, Perochon J, Lestrade L, Mahillon J, Chandler M. ISfinder: the reference centre for bacterial insertion sequences. Nucleic Acids Res. 2006;34:D32-D36. PMID: 16381877 .

71. Turton JF, Ward ME, Woodford $\mathrm{N}$ et al. The role of ISAba1 in expression of OXA carbapenemase genes in Acinetobacter baumannii. FEMS Microbiol Lett. 2006;258:72-77. doi: 10.1111/j.1574-6968.2006.00195.x.

72. Vranić-Ladavac M, Bedenić B, Minandri F et al. Carbapenem resistance and acquired class $\mathrm{D}$ beta-lactamases in Acinetobacter baumannii from Croatia 2009-2010. Eur J Clin Microbiol Infect Dis. 2014;33:471-478. doi: 10.1007/s10096013-1991-9.

73. Corvec S, Poirel L, Naas T, Drugeon H, Nordmann P. Genetics and expression of the carbapenem-hydrolyzing oxacillinase gene blaOXA-23 in Acinetobacter baumannii. Antimicrob Agents Chemother. 2007;51:1530-1533. doi: 10.1128/AAC.01132-06.

74. Poirel L, Nordmann P. Genetic structures at the origin of acquisition and expression of the carbapenem-hydrolyzing oxacillinase gene blaOXA-58 in Acinetobacter baumannii.Antimicrob Agents Chemother. 2006;50:1442-1448. doi: 10.1128/ AAC.50.4.1442-1448.2006.

75. Mugnier PD, Poirel L, Nordmann P. Functional analysis of insertion sequence IS $A b a 1$, responsible for genomic plasticity of Acinetobacter baumannii.J Bacteriol. 2009;191:2414-2418. doi: 10.1128/JB.01258-08.

76. Hall RM. Integrons and gene cassettes: hotspots of diversity in bacterial genomes. Ann N Y Acad Sci. 2012;1267:71-78. doi: 10.1111/j.1749-6632.2012.06588.x.

77. Gillings MR. Integrons: past, present, and future. Microbiol Mol Biol Rev. 2014;78:257-277. doi: 10.1128/MMBR.00056-13.

78. Turton JF, Kaufmann ME, Glover J et al. Detection and typing of integrons in epidemic strains of Acinetobacter baumannii found in the United Kingdom. J Clin Microbiol. 2005;43:3074-3082. doi: 10.1128/JCM.43.7.3074-3082.2005.

79. Peymani A, Farajnia S, Nahaei MR et al. Prevalence of class 1 integron among multidrug-resistant Acinetobacter baumannii 
in Tabriz, northwest of Iran. Pol J Microbiol. 2012;61:57-60. PMID: 22708347.

80. Martins N, Picão RC, Adams-Sapper S, Riley LW, Moreira BM. Association of class 1 and 2 integrons with multi-drug-resistant Acinetobacter baumannii international clones and Acinetobacter nosocomialis isolates. Antimicrob Agents Chemother. 2015;59:698-701. doi: 10.1128/AAC.02415-14.

81. Da Silva GJ, Correia M, Vital C et al. Molecular characterization of blaIMP-5, a new integron-borne metallo-beta-lactamase gene from an Acinetobacter baumannii nosocomial isolate in Portugal. FEMS Microbiol Lett. 2002;215:33-39. PMID: 12393197.

82. Huang LY, Chen TL, Lu PL et al. Dissemination of multi-drug-resistant, class 1 integron-carrying Acinetobacter baumannii isolates in Taiwan. Clin Microbiol Infect. 2008;14:1010 -1019. doi: 10.1111/j.1469-0691.2008.02077.x.

83. Lee K, Yum JH, Yong D et al. Novel acquired metallo-beta-lactamase gene, blaSIM-1, in a class 1 integron from Acinetobacter baumannii clinical isolates from Korea. Antimicrob Agents Chemother. 2005;49:4485-4491. doi: 10.1128/ AAC.49.11.4485-4491.2005.

84. Karah N, Sundsfjord A, Towner K, Samuelsen Ø. Insights into the global molecular epidemiology of carbapenem non-susceptible clones of Acinetobacter baumannii. Drug Resist Updat. 2012;15:237-247. doi: 10.1016/j.drup.2012.06.001.

85. Krizova L, Dijkshoorn L, Nemec A. Diversity and evolution of AbaR genomic resistance islands in Acinetobacter baumannii strains of European clone I. Antimicrob Agents Chemother. 2011;55:3201-3206. doi: 10.1128/AAC.00221-11.

86. Post V, White PA, Hall RM. Evolution of AbaR-type genomic resistance islands in multiply antibiotic-resistant Acinetobacter baumannii.J Antimicrob Chemother. 2010;65:1162-1170. doi: 10.1093/jac/dkq095.

87. Saule M, Samuelsen Ø, Dumpis U et al. Dissemination of a carbapenem-resistant Acinetobacter baumannii strain belonging to international clone II/sequence type 2 and harboring a novel AbaR4-like resistance island in Latvia. Antimicrob Agents Chemother. 2013;57:1069-1072. doi: 10.1128/ AAC.01783-12.

88. Fournier PE, Vallenet D, Barbe V et al. Comparative genomics of multidrug resistance in Acinetobacter baumannii. PLoS Genet. 2006;2:e7. doi: 10.1371/journal.pgen.002 0007.

89. Kim DH, Choi JY, Kim HW et al. Spread of carbapenem-resistant Acinetobacter baumannii global clone 2 in Asia and AbaR-type resistance islands. Antimicrob Agents Chemother. 2013;57:5239-5246. doi: 10.1128/AAC.00633-13.

90. Shintani M, Sanchez ZK, Kimbara K. Genomics of microbial plasmids: classification and identification based on replication and transfer systems and host taxonomy. Front Microbiol. 2015;6:242. doi: 10.3389/fmicb.2015.00242.

91. Plasmid definition. Scitable by Nature education Web site. 2015. Available at: http://www.nature.com/scitable/definition/ plasmid-plasmids-28. Accessed April 9, 2015.

92. Bertini A, Poirel L, Mugnier PD, Villa L, Nordmann P, Carattoli A. Characterization and PCR-based replicon typing of resistance plasmids in Acinetobacter baumannii. Antimicrob Agents Chemother. 2010;54:4168-4177. doi: 10.1128/ AAC.00542-10.

93. Towner KJ, Evans B, Villa L et al. Distribution of intrinsic plasmid replicase genes and their association with carbapenem-hydrolyzing class D beta-lactamase genes in European clinical isolates of Acinetobacter baumannii.Antimicrob Agents Chemother. 2011;55:2154-2159. doi: 10.1128/AAC.01661-10.
94. Lee K, Lim YS, Yong D, Yum JH, Chong Y. Evaluation of the Hodge test and the imipenem-EDTA double-disk synergy test for differentiating metallo-beta-lactamase-producing isolates of Pseudomonas spp. and Acinetobacter spp. J Clin Microbiol. 2003;41:4623-4629. doi: 10.1128/JCM.41.10.4623-4629.2003.

95. Etest MBL. bioMérieux Web site. 2015. Available at: http:// www.biomerieux.com. Accessed December 19, 2014.

96. Doi Y, Potoski BA, Adams-Haduch JM, Sidjabat HE, Pasculle AW, Paterson DL. Simple disk-based method for detection of Klebsiella pneumoniae carbapenemase-type beta-lactamase by use of a boronic acid compound. J Clin Microbiol. 2008;46:4083-4086. doi: 10.1128/JCM.01408-08.

97. Żabicka D, Baraniak A, Gniadkowski M, Hryniewicz W. Detection of carbapenemase - Recommendations 2013, The National Reference Centre for Antimicrobial Susceptibility Testing Web site. 2015. Available at: http://www.korld.edu. pl. Accessed December 15, 2014.

98. Nordmann P, Poirel L, Dortet L. Rapid detection of carbapenemase-producing Enterobacteriaceae. Emerg Infect Dis. 2012;18:1503-1507. doi: 10.3201/eid1809.120355.

99. Dortet L, Poirel L, Nordmann P. Rapid detection of carbapenemase-producing Pseudomonas spp. J Clin Microbiol. 2012;50:3773-3776. doi: 10.1128/JCM.01597-12.

100. Dortet L, Bréchard L, Poirel L, Nordmann P. Impact of the isolation medium for detection of carbapenemase-producing Enterobacteriaceae using an updated version of the Carba NP test. J Med Microbiol. 2014;63:772-776. doi: 10.1099/ jmm.0.071340-0.

101. Dortet L, Poirel L, Errera C, Nordmann P. CarbAcineto NP test for rapid detection of carbapenemase-producing Acinetobacter spp. J Clin Microbiol. 2014;52:2359-2364. doi: 10.1128/JCM.00594-14.

102. Pires J, Novais A, Peixe L. Blue-carba, an easy biochemical test for detection of diverse carbapenemase producers directly from bacterial cultures. J Clin Microbiol. 2013;51:4281-4283. doi: 10.1128/JCM.01634-13.

103. Rapid CARB Blue Kit. Rosco Diagnostica Web site. 2015. Available at: http://www.rosco.dk/. Accessed January 9, 2015.

104. Bonnin RA, Naas T, Poirel L, Nordmann P. Phenotypic, biochemical, and molecular techniques for detection of metallo- $\beta$-lactamase NDM in Acinetobacter baumannii. J Clin Microbiol. 2012;50:1419-1421. doi: 10.1128/JCM.06276-11.

105. Wieser A, Schneider L, Jung J, Schubert S. MALDI-TOF MS in microbiological diagnostics-identification of microorganisms and beyond (mini review). Appl Microbiol Biotechnol. 2012;93:965-974. doi: 10.1007/s00253-011-3783-4.

106. Kempf M, Bakour S, Flaudrops C et al. Rapid detection of carbapenem resistance in Acinetobacter baumannii using matrix-assisted laser desorption ionization-time of flight mass spectrometry. PLoS One. 2012;7:e31676. doi: 10.1371/ journal.pone.0031676.

107. Lupo A, Papp-Wallace KM, Sendi P, Bonomo RA, Endimiani A. Non-phenotypic tests to detect and characterize antibiotic resistance mechanisms in Enterobacteriaceae. Diagn Microbiol Infect Dis. 2013;77:179-194. doi: 10.1016/j. diagmicrobio.2013.06.001.

108. Woodford N, Ellington MJ, Coelho JM et al. Multiplex PCR for genes encoding prevalent OXA carbapenemases in Acinetobacter spp. Int J Antimicrob Agents. 2006;27:351-353. doi: 10.1016/j.ijantimicag.2006.01.004.

109. Poirel L, Walsh TR, Cuvillier V, Nordmann P. Multiplex PCR for detection of acquired carbapenemase genes. Diagn Microbiol Infect Dis. 2011;70:119-123. doi: 10.1016/j.diagmicrobio.2010.12.002. 
110. Eischeid AC. SYTO dyes and EvaGreen outperform SYBR Green in real-time PCR. BMC Res Notes. 2011;4:263. doi: 10.1186/1756-0500-4-263.

111. Pasanen T, Koskela S, Mero S et al. Rapid molecular characterization of Acinetobacter baumannii clones with rep-PCR and evaluation of carbapenemase genes by new multiplex PCR in Hospital District of Helsinki and Uusimaa. PLoS One. 2014;21:e85854. doi: 10.1371/journal.pone.0085854.

112. Huang XZ, Cash DM, Chahine MA, Nikolich MP, Craft DW. Development and validation of a multiplex TaqMan real-time PCR for rapid detection of genes encoding four types of class D carbapenemase in Acinetobacter baumannii. J Med Microbiol. 2012;61:1532-1537. doi: 10.1099/jmm.0.045823-0.

113. Solanki R, Vanjari L, Ede N, Gungi A, Soory A, Vemu L. Evaluation of LAMP assay using phenotypic tests and conventional PCR for detection of blaNDM-1 and blaKPC genes among carbapenem-resistant clinical Gram-negative isolates. $J$ Med Microbiol. 2013;62:1540-1544. doi: 10.1099/jmm.0.059907-0.

114. Liu W, Zou D, Li Y et al. Sensitive and rapid detection of the new Delhi metallo-beta-lactamase gene by loop-mediated isothermal amplification. J Clin Microbiol. 2012;50:1580-1585. doi: 10.1128/JCM.06647-11.
115. Microarrays definition. NCBI Probe Web site. 2015. Available at: http://www.ncbi.nlm.nih.gov/probe/docs/techmicroarray/. Accessed February 3, 2015.

116. Dally S, Lemuth K, Kaase M, Rupp S, Knabbe C, Weile J. DNA microarray for genotyping antibiotic resistance determinants in Acinetobacter baumannii clinical isolates. Antimicrob Agents Chemother. 2013;57:4761-4768. doi: 10.1128/ AAC.00863-13.

117. Cuzon G, Naas T, Bogaerts P, Glupczynski Y, Nordmann P. Probe ligation and real-time detection of KPC, OXA-48, VIM, IMP, and NDM carbapenemase genes. Diagn Microbiol Infect Dis. 2013;76:502-505. doi: 10.1016/j.diagmicrobio.2013.05.004.

118. Braun SD, Monecke S, Thürmer A et al. Rapid identification of carbapenemase genes in gram-negative bacteria with an oligonucleotide microarray-based assay. PLoS One. 2014;9:e102232. doi: 10.1371/journal.pone.0102232.

119. Mancini N, Infurnari L, Ghidoli N et al. Potential impact of a microarray-based nucleic acid assay for rapid detection of Gram-negative bacteria and resistance markers in positive blood cultures. J Clin Microbiol. 2014;52:1242-1245. doi: 10.1128/JCM.00142-14.

Submitted: 7 August, 2015

Accepted after reviews: 18 May, 2016

Available as AoP: 6 June, 2016 